\title{
Density Functional Theory Investigation of Nonlinear Optical Properties of T-Graphene Quantum Dots
}

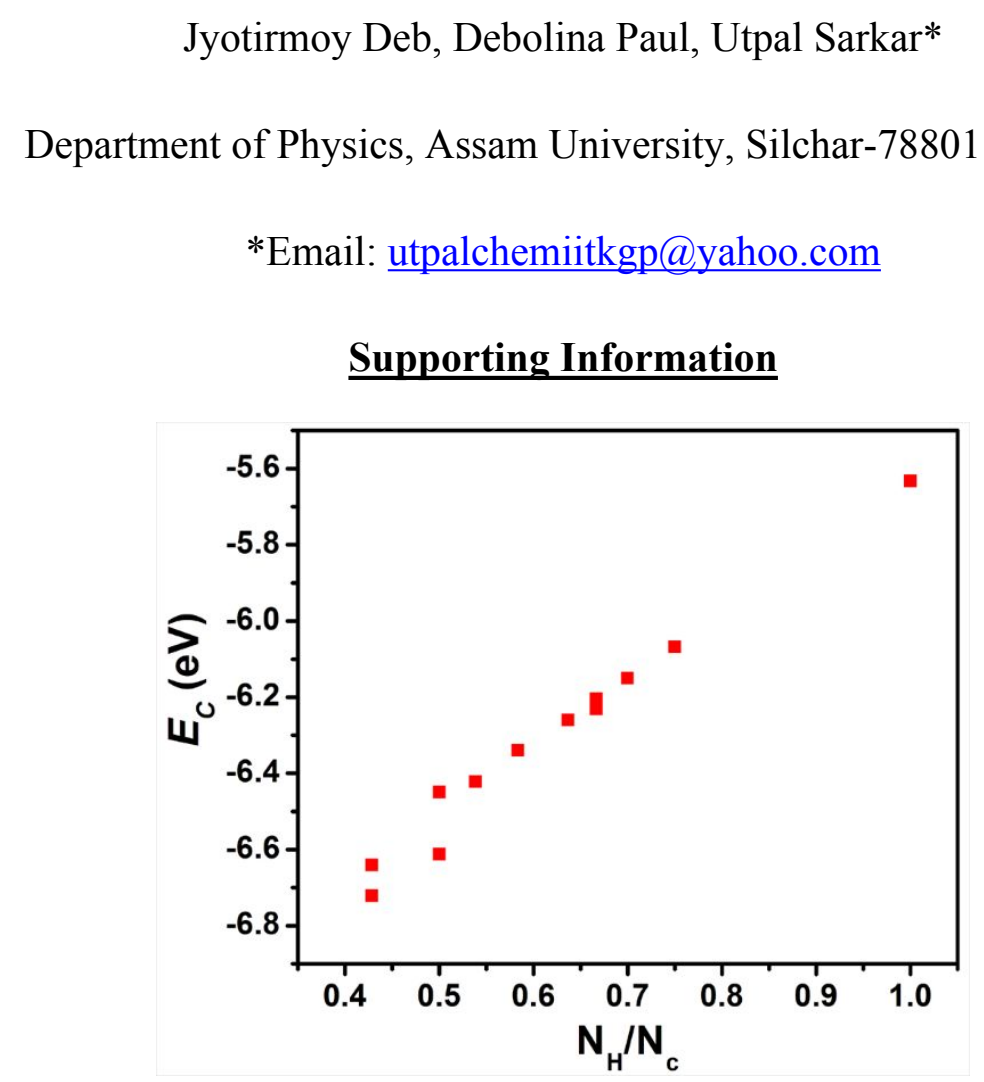

Figure S1. Variation of cohesive energy per atom $\left(E_{C}\right)$ as a function of 'the ratio of hydrogen atom to carbon atom $\left(\mathrm{N}_{\mathrm{H}} / \mathrm{N}_{\mathrm{C}}\right)^{\prime}$.

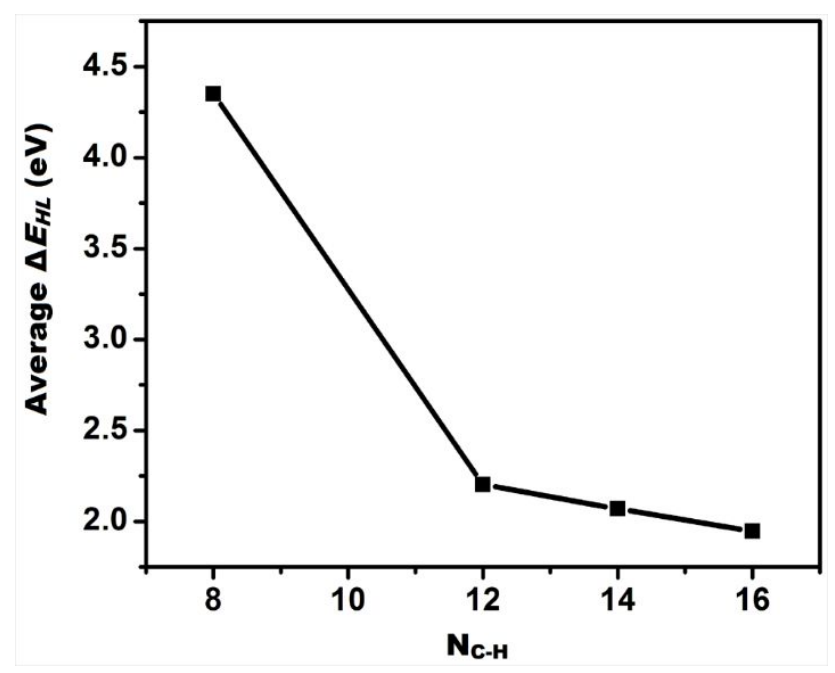

Figure S2. Variation of the average HOMO-LUMO gap (Average $\Delta E_{H L}$ ) with the number of edge carbon atoms connected to hydrogen atom $\left(\mathrm{N}_{\mathrm{C}-\mathrm{H}}\right)$. 
Table S1: Static and frequency dependent polarizabilities of T-GQDs

\begin{tabular}{|c|c|c|c|}
\hline \multirow{2}{*}{ Systems } & \multirow{\alpha}{*}{$(\mathbf{0} ; \mathbf{0})(\mathbf{a u})$} & \multicolumn{2}{|c|}{$\boldsymbol{\alpha ( \boldsymbol { \omega } ; \boldsymbol { ( \omega ) } ( \mathbf { a u } )}$} \\
\cline { 3 - 4 } & & $\omega=0.0340 \mathrm{au}$ & $\omega=0.0428 \mathrm{au}$ \\
\hline QD-1 & 0.00 & 0.00 & 0.00 \\
\hline QD-2 & 267.57 & 278.30 & 286.02 \\
\hline QD-3 & 0.00 & 0.00 & 0.00 \\
\hline QD-4 & 567.16 & 597.25 & 621.28 \\
\hline QD-5 & 205.68 & 209.00 & 211.04 \\
\hline QD-6 & 391.25 & 399.44 & 404.54 \\
\hline QD-7 & 327.40 & 340.07 & 349.08 \\
\hline QD-8 & 358.01 & 372.62 & 374.28 \\
\hline QD-9 & 363.09 & 375.51 & 384.13 \\
\hline QD-10 & 417.08 & 434.51 & 446.84 \\
\hline QD-11 & 0.00 & 0.00 & 0.00 \\
\hline QD-12 & 385.85 & 405.23 & 435.01 \\
\hline
\end{tabular}

Table S2: First order static and frequency dependent hyperpolarizabilities of T-GQDs

\begin{tabular}{|c|c|c|c|c|c|}
\hline Systems & $\beta(\mathbf{0} ; \mathbf{0 , 0})(\mathbf{a u})$ & \multicolumn{2}{|c|}{$\beta(-\omega ; \omega, 0)(\mathrm{au})$} & \multicolumn{2}{|c|}{$\beta(-2 \omega ; \omega, \omega)(\mathrm{au})$} \\
\hline & & $\omega=0.0340 \mathrm{au}$ & $\omega=0.0428 \mathrm{au}$ & $\omega=0.0340 \mathrm{au}$ & $\omega=0.0428 \mathrm{au}$ \\
\hline QD-1 & 0.00 & 0.00 & 0.00 & 0.00 & 0.00 \\
\hline QD-2 & 0.26 & 0.38 & 0.51 & 1.38 & 0.18 \\
\hline QD-3 & 0.00 & 0.00 & 0.00 & 0.00 & 0.00 \\
\hline QD-4 & 6.91 & 10.96 & 15.77 & 30.94 & 4.28 \\
\hline QD-5 & 0.01 & 0.01 & 0.01 & 0.01 & 0.01 \\
\hline QD-6 & 0.03 & 0.03 & 0.03 & 0.03 & 0.01 \\
\hline QD-7 & 81.51 & 191.31 & 321.25 & 909.69 & 1235.38 \\
\hline QD-8 & 145.21 & 156.94 & 88.38 & 611.40 & 29635.74 \\
\hline QD-9 & 256.25 & 358.54 & 445.85 & 1423.35 & 30353.07 \\
\hline QD-10 & 777.30 & 1022.19 & 1249.18 & 1467.33 & 2037.00 \\
\hline QD-11 & 0.00 & 0.00 & 0.00 & 0.00 & 0.00 \\
\hline QD-12 & 0.02 & 0.02 & 0.18 & 0.01 & 0.56 \\
\hline
\end{tabular}

Table S3: Second order static and frequency dependent hyperpolarizabilities of T-GQDs

\begin{tabular}{|c|c|c|c|c|c|}
\hline Systems & $\begin{array}{c}\gamma(\mathbf{0 ; 0 , 0 , 0 )}(\mathbf{a u}) \\
\times \mathbf{1 0}^{\mathbf{4}}\end{array}$ & \multicolumn{2}{|c|}{$\left.\gamma(-\boldsymbol{\omega} ; \boldsymbol{\omega}, \mathbf{0 , 0}) \times \mathbf{1 0}^{\mathbf{4}} \mathbf{( a u}\right)$} & \multicolumn{2}{c|}{$\gamma(-\mathbf{2} \omega ; \boldsymbol{\omega}, \boldsymbol{\omega , 0}) \times \mathbf{1 0}^{\mathbf{4}}(\mathbf{a u})$} \\
\hline & & $\omega=0.0340 \mathrm{au}$ & $\omega=0.0428 \mathrm{au}$ & $\omega=0.0340 \mathrm{au}$ & $\omega=0.0428 \mathrm{au}$ \\
\hline QD-1 & 0.00 & 0.00 & 0.00 & 0.00 & 0.00 \\
\hline QD-2 & 5.80 & 5.88 & 5.47 & 16.84 & 11.87 \\
\hline QD-3 & 0.00 & 0.00 & 0.00 & 0.00 & 0.00 \\
\hline QD-4 & 10.08 & 12.78 & 17.95 & 20.37 & 68.52 \\
\hline
\end{tabular}




\begin{tabular}{|c|c|c|c|c|c|}
\hline QD-5 & 8.23 & 9.02 & 9.40 & 10.47 & 12.15 \\
\hline QD-6 & 22.30 & 24.97 & 26.52 & 30.87 & 39.65 \\
\hline QD-7 & 7.31 & 8.42 & 9.08 & 37.43 & 17.67 \\
\hline QD-8 & 13.15 & 16.59 & 383.41 & 16.41 & 116.70 \\
\hline QD-9 & 29.27 & 36.63 & 44.44 & 52.35 & 31.47 \\
\hline QD-10 & 41.16 & 52.85 & 64.37 & 83.15 & -14.84 \\
\hline QD-11 & 0.00 & 0.00 & 0.00 & 0.00 & 0.00 \\
\hline QD-12 & 8.76 & 10.71 & 27.94 & 73.33 & 831.25 \\
\hline
\end{tabular}

Table S4: Nucleus-independent chemical shifts (NICS) (ppm) for the sets of four and eight membered T-GQDs ring systems

\begin{tabular}{|c|c|c|}
\hline System & Four membered ring & Eight membered ring \\
\hline QD-1 & --- & 4.7480 \\
\hline QD-2 & -15.5550 & $16.5465,16.5491$ \\
\hline QD-3 & $-15.9927,-15.9928$ & $8.7541,13.7745,8.7539$ \\
\hline QD-4 & $\begin{array}{l}-15.1475,-15.1477,-15.1217,- \\
15.1207\end{array}$ & $\begin{array}{l}-5.9548,-5.9633,-5.9647 \\
5.9575,-34.0643\end{array}$ \\
\hline QD-5 & $\begin{array}{lll}5.8034, & 5.8035, & 5.8033, \\
5.8038 & & \end{array}$ & 0.6719 \\
\hline QD-6 & $\begin{array}{lcr}7.5383, & 5.3951, & 7.5383, \\
7.5383, & 5.3951,7.5383,4.5579 \\
\end{array}$ & $0.4468,0.4468$ \\
\hline QD-7 & -22.0991 & $-2.7811,-2.7811,-18.1144$ \\
\hline QD-8 & $-14.8682, \quad 29.3552$ & $14.0344,4.0297,-4.6604$ \\
\hline QD-9 & $1.9918,-9.7934,-5.6578$ & $7.0796,-0.1718,-1.2938$ \\
\hline QD-10 & $\begin{array}{lll}-4.7506, & -5.0487, & -5.0475, \\
2.1215 & & \\
\end{array}$ & $0.7109,0.1022,0.1027$ \\
\hline QD-11 & $\begin{array}{lll}32.1087, & 32.1087, & 32.1088, \\
23.4915, & 32.1087\end{array}$ & $\begin{array}{l}23.6395,24.5845, \quad 24.5845, \\
23.6395\end{array}$ \\
\hline QD-12 & -29.3391 & $\begin{array}{l}-7.1668,-7.1546,-7.1546, \\
7.1668\end{array}$ \\
\hline
\end{tabular}

